\title{
Guía de la EAU sobre el cáncer de próstata. Parte I: cribado, diagnóstico y tratamiento del cáncer clínicamente localizado ${ }^{\text {is }}$
}

\author{
A. Heidenreich ${ }^{a, *}$, J. Bellmunt ${ }^{b}$, M. Bolla ${ }^{c}$, S. Joniau ${ }^{d}$, M. Mason ${ }^{e}$, V. Matveev ${ }^{f}$, \\ N. Mottet $^{\text {g }}$, H.P. Schmid ${ }^{\mathrm{h}}$, T. van der Kwast ${ }^{\mathrm{i}}$, T. Wiegel ${ }^{\mathrm{j}}$ y F. Zattoni ${ }^{\mathrm{k}}$
}

a Departamento de Urología, Universidad RWTH Aachen, Aachen, Alemania

b Departamento de Oncología Médica, Hospital Universitario del Mar, Barcelona, España

c Departamento de Radioterapia, C.H.U. Grenoble, Grenoble, Francia

d Departamento de Urología, Hospital Universitario, Lovaina, Bélgica

e Departamento de Oncología y Medicina Paliativa, Velindre Hospital, Cardiff, Reino Unido

f Departamento de Urología, Academia Rusa de Ciencia Médica, Centro de Investigación del Cáncer, Moscú, Rusia

s Departamento de Urología, Clinique Mutualiste de la Loire, Saint Etienne, Francia

h Departamento de Urología, Kantonspital St. Gallen, San Gallen, Suiza

' Departamento de Patología, Red Universitaria de Salud, Toronto, Canadá

j Departamento de Oncología Radioterápica, Hospital Universitario Ulm, Ulm, Alemania

k Departamento de Urología, Universidad de Padua, Padua, Italia

Recibido el 11 de marzo de 2011; aceptado el 11 de marzo de 2011

Accesible en línea el 14 de julio de 2011

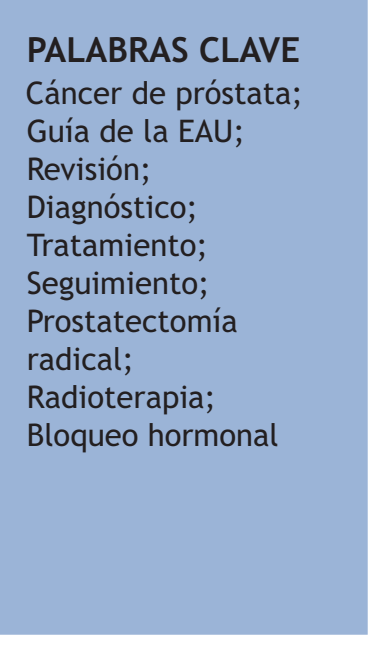

\begin{abstract}
Resumen
Objetivo: Nuestro objetivo era presentar un resumen de la versión de 2010 de la guía de la Asociación Europea de Urología (EAU) para el cribado, diagnóstico y tratamiento del cáncer de próstata (CP) clínicamente localizado.

Métodos: El grupo de trabajo realizó una revisión de los nuevos datos aparecidos desde 2007 hasta 2010 en la literatura. Las guías se actualizaron y el nivel de evidencia y grado de recomendación se incorporaron al texto basándose en una revisión sistemática de la literatura, que incluía una búsqueda en las bases de datos en línea y revisiones bibliográficas.

Resultados: Existe una versión completa disponible en las oficinas o el sitio web de la EAU (www.uroweb.org). Los indicios actuales son insuficientes para garantizar un cribado extendido del conjunto de la población mediante el antígeno prostático específico (APE) para el CP. El método diagnóstico de elección es la biopsia sistematizada de la próstata bajo control ecográfico con anestesia local. El seguimiento activo representa una opción viable en varones con CP de bajo riesgo y una larga esperanza de vida. Un tiempo de duplicación del APE $<3$ años, o una progresión en la biopsia, indicaría la necesidad de intervención activa. En varones con CP localmente avanzado en los que la terapia local no sea obligatoria, la espera en observación
\end{abstract}

\footnotetext{
论 La traducción de este artículo se ha llevado a cabo con el permiso de la Asociación Europea de Urología.

* Autor para correspondencia.

Correo electrónico: aheidenreich@ukaachen.de (A. Heidenreich).
} 


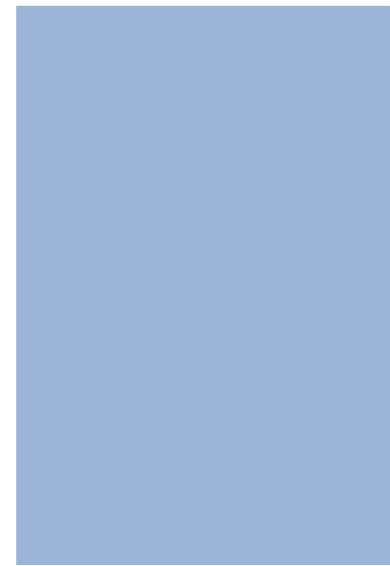

\section{KEYWORDS}

Prostate cancer;

EAU guidelines;

Review;

Diagnosis;

Treatment;

Follow-up;

Radical

prostatectomy;

Radiation therapy;

Androgen deprivation (watchful waiting [WW]) es un tratamiento alternativo a la terapia de bloqueo hormonal o de privación androgénica (TPA), con una eficacia oncológica equivalente. El tratamiento activo se recomienda en la mayoría de los pacientes con enfermedad localizada y una larga esperanza de vida, siendo la prostatectomía radical (PR) superior a la WW en un ensayo aleatorio prospectivo. La PR con conservación nerviosa es la técnica de elección en la enfermedad limitada al órgano; el bloqueo hormonal neoadyuvante no ha demostrado una mejoría en las variables de resultados. La radioterapia debe realizarse con al menos $74 \mathrm{~Gy}$ en el CP de bajo riesgo y con $78 \mathrm{~Gy}$ en el que presenta un riesgo intermedio/alto. Para casos en los que la enfermedad esté localmente avanzada la TPA adyuvante durante tres años logra tasas de supervivencia superiores a nivel general y específico de la enfermedad, y supone el tratamiento preferido. El seguimiento tras terapia local se basa principalmente en los niveles de APE, estando la anamnesis específica de la enfermedad con diagnóstico por imagen sólo indicada cuando aparecen los síntomas.

Conclusiones: Lo que se conoce acerca del campo del CP está cambiando rápidamente. Esta guía de la EAU para el CP resume los hallazgos más recientes y los aplica a la práctica clínica. (C) 2011 AEU. Publicado por Elsevier España, S.L. Todos los derechos reservados.

\section{EAU Guidelines on Prostate Cancer. P5art I: Screening, Diagnosis, and Treatment of Clinically Localised Disease}

\section{Abstract}

Objective: Our aim was to present a summary of the 2010 version of the European Association of Urology (EAU) guidelines on the screening, diagnosis, and treatment of clinically localised cancer of the prostate (PCa).

Methods: The working panel performed a literature review of the new data emerging from 2007 to 2010. The guidelines were updated, and level of evidence and grade of recommendation were added to the text based on a systematic review of the literature, which included a search of online databases and bibliographic reviews.

Results: A full version is available at the EAU office or Web site (www.uroweb.org). Current evidence is insufficient to warrant widespread population-based screening by prostate-specific antigen (PSA) for PCa. A systematic prostate biopsy under ultrasound guidance and local anaesthesia is the preferred diagnostic method. Active surveillance represents a viable option in men with low-risk PCa and a long life expectancy. PSA doubling time in $<3 \mathrm{yr}$ or a biopsy progression indicates the need for active intervention. In men with locally advanced PCa in whom local therapy is not mandatory, watchful waiting (WW) is a treatment alternative to androgen-deprivation therapy (ADT) with equivalent oncologic efficacy. Active treatment is mostly recommended for patients with localised disease and a long life expectancy with radical prostatectomy (RP) shown to be superior to WW in a prospective randomised trial. Nervesparing RP represents the approach of choice in organ-confined disease; neoadjuvant androgen deprivation demonstrates no improvement of outcome variables. Radiation therapy should be performed with at least $74 \mathrm{~Gy}$ and $78 \mathrm{~Gy}$ in low-risk and intermediate/high-risk PCa, respectively. For locally advanced disease, adjuvant ADT for 3 yr results in superior disease-specific and overall survival rates and represents the treatment of choice. Follow-up after local therapy is largely based on PSA, and a disease-specific history with imaging is indicated only when symptoms occur.

Conclusions: The knowledge in the field of PCa is rapidly changing. These EAU guidelines on $\mathrm{PCa}$ summarise the most recent findings and put them into clinical practice.

(C) 2011 AEU. Published by Elsevier España, S.L. All rights reserved.

\section{Introducción}

El resumen más reciente de la guía de la Asociación Europea de Urología (EAU) para el cáncer de próstata (CP) se publicó en $2008^{1}$. La versión ampliada de esta guía está actualizándose continuamente debido a los importantes cambios que se han producido y que afectan al tratamiento clínico del CP. Este documento resume la actualización de 2010 de la guía de la EAU para el CP. Para facilitar la evaluación de la calidad de la información suministrada se han incluido el nivel de evidencia y el grado de recomendación de acuerdo con los principios generales de la medicina actual $^{2}$.

\section{Epidemiología}

En Europa el CP es la neoplasia sólida más común, con una tasa de incidencia de 214 casos por cada 1.000 varones, lo que supera al cáncer de pulmón y colorrectal ${ }^{3}$. El CP afecta más frecuentemente a los varones de mayor edad, por lo que 
constituye una importante preocupación en cuanto a la salud en los países desarrollados. Así, aproximadamente el 15\% de los cánceres en varones son $\mathrm{CP}$ en países desarrollados, en comparación con el $4 \%$ de los casos de cáncer en varones en países en vías de desarrollo ${ }^{4}$. Existen grandes diferencias regionales en las tasas de incidencia del $C P$, que varía de los 68,8 de Malta a 182 de Bélgica ${ }^{4}$.

\section{Factores de riesgo}

Los factores que determinan el riesgo de desarrollar un CP clínico no se conocen con precisión, aunque se han identificado tres factores de riesgo bien definidos: edad avanzada, etnia y factores hereditarios.

Si un familiar de primer grado tiene la enfermedad, como mínimo el riesgo se duplica. Si dos o más familiares de primer grado están afectados el riesgo se incrementa entre 5 y 11 veces $^{5}$. Aproximadamente el $9 \%$ de los individuos con $\mathrm{CP}$ padece de CP hereditario genuino, definido como tres o más familiares afectados o como mínimo dos familiares que hayan desarrollado precozmente la enfermedad (es decir $<55$ años).

\section{Clasificaciones}

En esta guía se utiliza la clasificación TNM de 2009 de la Unión Internacional Contra el Cáncer ${ }^{6}$. El método recomendado para la clasificación del CP es el grado de Gleason. De acuerdo con la convención internacional actual, el grado (modificado) de Gleason para cánceres detectados en una biopsia de próstata se compone del grado de Gleason del carcinoma dominante (más extendido) más el grado más elevado, independientemente de su extensión (sin la regla del $5 \%)^{7}$. En muestras de prostatectomía radical (PR) debe indicarse tanto el grado primario como el secundario en la escala de Gleason. También debe indicarse la presencia de un grado terciario y su proporción aproximada respecto al volumen del cáncer.

\section{Cribado del cáncer de próstata}

Actualmente no se dispone de indicios suficientes para la instauración de programas de cribado del total de la población para la detección precoz del CP en todos los varones ${ }^{8}$ (nivel de evidencia: 2). Para evaluar la eficacia del cribado del CP se han publicado dos grandes ensayos aleatorios: el Ensayo para cáncer de próstata, de pulmón, colorrectal y de ovario (Prostate, Lung, Colorectal, and Ovary [PLCO]) en EE. UU. y el Estudio Europeo Aleatorizado de Detección de Cáncer de Próstata (European Randomised Study of Screening for Prostate Cancer [ERSPC]) en Europa9,10 (nivel de evidencia: $1 \mathrm{~b})$.

El ensayo PLCO realizó una selección y posterior distribución aleatoria de 76.693 varones para recibir un cribado anual mediante análisis del antígeno prostático específico (APE) y tacto rectal o atención estándar como control ${ }^{9}$. Tras un seguimiento de 7 años la incidencia del CP por cada 10.000 personas-año fue de 116 (2.820 cánceres) en el grupo de cribado y de 95 (2.322 cánceres) en el grupo de control (cociente de tasas: 1,22) ${ }^{9}$. La incidencia de fallecimientos por cada 10.000 personas-año fue de 2,0 (50 fallecimientos) en el grupo de cribado y de 1,7 (44 fallecimientos) en el grupo de control (cociente de tasas: 1,13). El equipo del proyecto PLCO llegó a la conclusión de que la mortalidad por $\mathrm{CP}$ en individuos con cáncer detectado mediante cribado fue muy baja, y no presentaba diferencias significativas entre los dos grupos de estudio (nivel de evidencia: $1 \mathrm{~b})$.

El estudio ERSPC incluyó un total de 162.243 varones de entre 55 y 69 años $^{10}$. Se les asignó aleatoriamente a un grupo con cribado mediante APE cada 4 años, de media, o a un grupo de control sin cribado. Durante un seguimiento de 9 años de media la incidencia acumulada del CP fue del $8,2 \%$ en el grupo de cribado y del $4,8 \%$ en el grupo de control ${ }^{10}$. La diferencia absoluta de riesgo fue de 0,71 por 1.000 varones. Esto significa que debería cribarse a 1.410 varones y deberían tratarse 48 casos de CP adicionales para evitar un fallecimiento por cáncer. Los investigadores del estudio ERSPC llegaron a la conclusión de que el cribado basado en APE reduciría la tasa de fallecimientos por CP en un $20 \%$, pero se asociaba a un alto riesgo de tratamiento excesivo (nivel de evidencia: $1 \mathrm{~b}$ ).

Ambos ensayos han sido objeto de enorme atención, siendo muy comentados. En el ensayo PLCO la tasa de cumplimiento en el grupo de estudio de cribado fue del $85 \%$ en las pruebas de APE, y del $86 \%$ en el caso de tacto rectal. Sin embargo, la tasa de contaminación en el grupo de estudio de control fue muy elevada, llegando al $40 \%$ el primer año, aumentando hasta el $52 \%$ el sexto año la adecuación de las pruebas de APE y variando del 41 al $46 \%$ en el tacto rectal. Es más, el cumplimiento de la biopsia fue sólo del $40-52 \%$ frente al $86 \%$ en el estudio ERSPC. Por lo tanto, es posible que el ensayo PLCO nunca pueda determinar si el cribado puede influir en la mortalidad por CP.

En un reciente análisis retrospectivo de la incidencia del CP se evaluaron la metástasis del CP y la causa del fallecimiento en un grupo de 11.970 varones incluidos en el grupo de estudio de intervención del estudio ERSPC, y una población de control de 133.287 varones no sometidos a cribado durante un periodo de observación de 8 años ${ }^{11}$. El riesgo relativo (RR) de metástasis del $\mathrm{CP}$ en la población sometida a cribado, en comparación con la población de control, fue $0,47(p<0,001)$. El RR de mortalidad específica por CP también fue significativamente bajo en el grupo de estudio de cribado (RR: 0,63; $p=0,008$ ). La reducción absoluta de la mortalidad fue de 1,8 fallecimientos por 1.000 varones. Basándose en esos datos, las verdaderas ventajas del estudio ERSPC sólo serán evidentes tras 10-15 años de seguimiento, especialmente por el impacto que tendrá la reducción del $41 \%$ en la metástasis del grupo de estudio de cribado. Además, es preciso esperar a los resultados relativos a la carga económica y a los efectos secundarios resultantes de un cribado más intensivo.

Basándose en los resultados de estos dos grandes ensayos aleatorios, la mayoría, si no todas las principales sociedades urológicas, han llegado a la conclusión de que, en la actualidad, el cribado de grandes poblaciones para el CP no es conveniente. En su lugar, debería ofrecerse una detección precoz (cribado oportunista) a pacientes bien informados (consultar la sección 6). Aún quedarían abiertas dos preguntas con carácter empírico: 
Tabla 1 Riesgo de cáncer de próstata en relación con valores reducidos del antígeno prostático específico

\begin{tabular}{lc}
\hline Nivel de APE $(\mathrm{ng} / \mathrm{ml})$ & Riesgo de CP $(\%)$ \\
\hline $0-0,5$ & 6,6 \\
$0,6-1$ & 10,1 \\
$1,1-2$ & 17,0 \\
$2,1-3$ & 23,9 \\
$3,1-4$ & 26,9 \\
\hline
\end{tabular}

APE: antígeno prostático específico; CP: cáncer de próstata.

1. ¿A qué edad debería iniciarse la detección precoz?

2. ¿Cuál sería el intervalo para las pruebas de APE y el tacto rectal?

La decisión de someterse a pruebas precoces de APE debería consensuarse entre el paciente y el médico, teniendo en cuenta la información disponible y las distintas ventajas e inconvenientes. Se ha sugerido una determinación inicial del APE a los 40 años, sobre la que podría basarse el intervalo de cribado posterior ${ }^{10-12}$ (grado de recomendación: B). Un intervalo de cribado de 8 años podría ser suficiente en varones con niveles iniciales de $A P E \leq 1$ $\mathrm{ng} / \mathrm{ml}^{10}$. No se haría necesario realizar pruebas de APE adicionales en varones $>75$ años y con un APE inicial $\leq 3 \mathrm{ng} / \mathrm{ml}$ debido al riesgo extremadamente bajo de mortalidad por $C P^{13}$.

\section{Diagnóstico y estadificación del cáncer de próstata}

Las principales herramientas diagnósticas para detectar la existencia de un CP incluyen el tacto rectal, la concentración sérica de APE y las biopsias transrectales ecodirigidas (BTE). En aproximadamente el 18\% del total de pacientes el $\mathrm{CP}$ se detecta únicamente mediante un tacto rectal por cuyo resultado se sospeche la presencia de cáncer, independientemente del nivel de APE ${ }^{14}$ (nivel de evidencia: $2 a$ ). Un tacto rectal por cuyo resultado se sospeche la presencia de cáncer en pacientes con un nivel de APE de hasta $2 \mathrm{ng} / \mathrm{ml}$ tiene el valor diagnóstico de un resultado positivo del $5-30 \%{ }^{15}$ (nivel de evidencia: $2 a$ ).

Es necesario definir el umbral de APE que indique el mayor riesgo de CP (tabla 1). Al analizar los niveles séricos de APE es preciso tener en cuenta que no todos los análisis de APE darán como resultado la misma concentración sérica ${ }^{16}$. Por lo tanto, la situación en la que se encuentra la interpretación clínica de los resultados de APE o el porcentaje de APE libre (APEl) es complicada. La comparación metodológica entre los ensayos tradicionales de calibración de APE y APEl con Hybritech, y por los nuevos ensayos de calibración con Access que representan el estándar para la Organización Mundial de la Salud (OMS), produjo resultados un 25\% más bajos aproximadamente para el APE y el APEl. Debe considerarse un umbral de APE de 3 o 3,1 $\mu \mathrm{g} / \mathrm{l}$ para los ensayos de calibración de la OMS para lograr el mismo perfil de sensibilidad/especificidad que con un umbral de $4 \mu \mathrm{g} / \mathrm{l}$ de los ensayos tradicionales de calibración. Los umbrales del porcentaje de APEl pueden mantenerse.

El nivel de APE es un parámetro continuo; cuanto mayor sea el valor, mayor será la probabilidad de existencia de cáncer. El descubrimiento de que muchos varones podrían albergar un CP, a pesar de valores bajos de APE sérico, ha sido subrayado por los resultados de un estudio de prevención realizado en $E E$. UU. recientemente ${ }^{17}$ (nivel de evidencia: $2 \mathrm{a}$ ). La tabla 2 indica la tasa de CP en relación con el APE sérico de 2.950 varones del grupo de estudio del placebo y con valores normales de APE.

Se han descrito diversas modificaciones en el valor del APE sérico que pueden mejorar la especificidad del APE para la detección precoz del CP; entre otras estarían la densidad del APE, la densidad del APE de la zona de transición, los intervalos de referencia específicos de la edad y las formas moleculares del APE.

En un ensayo prospectivo multicéntrico el CP se detectó mediante biopsia en el $56 \%$ de los varones con un APE libre/total (APEl/APEt) < 0,10, pero sólo en el $8 \%$ de los varones con un APEl/APEt $>0,25^{8}$ (nivel de evidencia: $2 a$ ). Estos datos se confirmaron en una reciente prueba de cribado sobre 27.730 varones con una concentración sérica de APE de entre 2,1 y $10 \mathrm{ng} / \mathrm{ml}^{18}$. Mediante el uso de APEl/APEt el número de biopsias innecesarias se redujo de forma significativa y la tasa de detección del CP aumentó también significativamente. No obstante, el concepto debe utilizarse con precaución, ya que existen diversos factores preanalíticos y clínicos que podrían influir en el APEL/APEt. Por ejemplo, el APEl es inestable tanto a $4{ }^{\circ} \mathrm{C}$ como a temperatura ambiente.

Los dos conceptos de velocidad del APE (VAPE) y tiempo de duplicación del APE (DT APE) tienen un uso limitado en el diagnóstico del CP debido a varias cuestiones sin resolver, entre las que se incluyen el ruido de fondo (volumen total de la próstata, hiperplasia prostática benigna), el intervalo entre determinaciones de APE y la aceleración/desaceleración de la velocidad y el tiempo de duplicación del APE con el paso del tiempo. Los estudios prospectivos no han demostrado que estas medidas puedan proporcionar información adicional en comparación con el APE aislado ${ }^{19,20}$.

A diferencia de los marcadores séricos mencionados anteriormente, el nuevo biomarcador antígeno de cáncer de próstata 3 (ACP3) se mide en el sedimento urinario obtenido tras un masaje prostático ${ }^{21}$. La determinación de este ARN específico del CP es experimental. A escala poblacional parece ser de utilidad, pero su impacto en el paciente individual sigue siendo altamente cuestionable. Hasta ahora, ninguno de los biomarcadores mencionados puede utilizarse para aconsejar a un paciente individual sobre la necesidad de realizar una biopsia de próstata para descartar el CP. El marcador molecular podría ayudar en el proceso de toma de decisiones en lo relativo a una posible repetición de la biopsia en varones con una primera biopsia con resultado negativo, pero con sospecha persistente de CP2,23. Los varones con biopsia de seguimiento con resultado positivo presentaron puntuaciones de ACP3 significativamente mayores en comparación con los varones con una segunda biopsia con resultado negativo $(69,5$ frente a 37,$7 ; p<0,001)$. En varones con un APEl/APEt < 10\%, la puntuación de ACP3 se identificó como un factor pronóstico significativo del CP. Sin embargo, en varones con un APEL/APEt del $10-20 \%$ y $>20 \%$ el porcentaje de biopsias positivas se incrementaba del 17,8 al $30,6 \%$ y del 23,9 al $37 \%$, respectivamente, si se utilizaba una puntuación de ACP3 > 30 . 
Tabla 2 Orientaciones y recomendaciones para la prostatectomía radical (a) y la radioterapia definitiva (b)

\section{(a)}

Indicaciones

Nivel de evidencia

Pacientes con CP localizado de riesgo bajo e intermedio (cT1a-T2b, grado de Gleason 2-7 y APE

$1 \mathrm{~b}$

$\leq 20)$ y una esperanza de vida $>10$ años

Opcional

Pacientes en el estadio T1a y una esperanza de vida >15 años o grado de Gleason 7

Pacientes seleccionados con CP localizado de alto riesgo y bajo volumen (cT3a o grado de

Gleason 8-10 o APE > 20)

Pacientes muy seleccionados con CP localizado de riesgo muy alto (cT3b-T4 NO o cualquier T

$\mathrm{N} 1)$ en el contexto de un tratamiento multimodal

No es aconsejable la terapia neoadyuvante a corto ( 3 meses) o a largo plazo ( 9 meses) con análogos de la hormona liberadora de gonadotropina en el tratamiento de la enfermedad en el estadio T1-T2

Se debería intentar realizar una intervención quirúrgica con conservación nerviosa en

pacientes con potencia preoperatoria que presenten bajo riesgo de enfermedad extracapsular

(T1c, grado de Gleason $<7$ y APE $<10 \mathrm{ng} / \mathrm{ml}$ ).

Los procedimientos con conservación nerviosa unilateral son otra opción para la enfermedad

Recomendaciones

en el estadio clínico T2a-c

(b)

Indicaciones

En el caso de pacientes del grupo de alto riesgo, una TPA a corto plazo antes y durante la

radioterapia da como resultado un aumento de la tasa de supervivencia general

La braquiterapia intersticial transperineal con implantes permanentes es otra opción para los pacientes con $\mathrm{CT} 1-\mathrm{T} 2 \mathrm{a}$, grado de Gleason $<7, \mathrm{APE} \leq 10 \mathrm{ng} / \mathrm{ml}$, volumen prostático $\leq 50 \mathrm{ml}$ y un buen baremo internacional de síntomas prostáticos y sin una RTUP previa

La irradiación externa postoperatoria inmediata tras una PR en pacientes con un tumor patológico en el estadio T3 NO MO mejora la supervivencia bioquímica y clínica sin la enfermedad

La irradiación externa postoperatoria inmediata tras una PR en pacientes con márgenes quirúrgicos positivos mejora la supervivencia bioquímica y clínica sin la enfermedad

Una opción alternativa es aplicar radiación en el momento que se produzca un fallo bioquímico, pero antes de que el APE supere los $0,5 \mathrm{ng} / \mathrm{ml}$

En un CP localmente avanzado T3-4 NO MO y en una enfermedad de alto riesgo, la tasa de supervivencia general mejora a través de una terapia hormonal concomitante y adyuvante con una duración total de tres años, en la que se aplica una irradiación externa en pacientes con un estado de rendimiento 0-2 de la OMS

En un subconjunto de pacientes con T2c-T3 N0-x y grado de Gleason 6, una TPA a corto plazo antes y durante la radioterapia puede influir favorablemente en la tasa de supervivencia general.

APE: antígeno prostático específico; CP: cáncer de próstata; OMS: Organización Mundial de la Salud; PR: prostatectomía radical; RIM: radioterapia de intensidad modulada; RTC3D: radioterapia conformada en 3D; RTUP: resección transureteral de la próstata; TPA: tratamiento de deprivación androgénica.

La BTE, o la biopsia transperineal dirigida lateralmente con aguja gruesa de calibre $18 \mathrm{G}$, se ha convertido en el método estándar para la obtención de material para exámenes histopatológicos ${ }^{24,25}$. La necesidad de biopsias de próstata debería determinarse basándose en el nivel de APE y un tacto rectal que haga sospechar de la presencia de cáncer. La edad biológica del paciente, las posibles comorbilidades y las consecuencias terapéuticas también deberían tenerse en cuenta. El primer nivel elevado de APE no debería suponer biopsia inmediata, pero debería verificarse tras un par de semanas mediante el mismo ensayo bajo condiciones estándar, excepto en el caso de valores de APE elevados $>20 \mathrm{ng} / \mathrm{ml}$ una vez excluida la prostatitis.
Con un volumen glandular de $30-40 \mathrm{ml}$ deberían tomarse al menos 8 muestras. Más de 12 muestras no resultarán más significativas $^{26}$ (nivel de evidencia: 1a). Las quinolonas orales o intravenosas son antibióticos preventivos novedosos con ciprofloxacina, que es superior a la ofloxacina ${ }^{27}$ (nivel de evidencia: 1b). El bloqueo periprostático ecodirigido es un método novedoso ${ }^{28}$ (nivel de evidencia: $1 \mathrm{~b}$ ). En las biopsias iniciales las muestras deberían tomarse lo más cerca posible de la parte posterior y lateral de la glándula periférica. Deben obtenerse muestras adicionales de zonas donde se sospeche la presencia de cáncer mediante tacto rectal/BTE.

Las indicaciones para repetir las biopsias serían un nivel en aumento $\mathrm{y} / \mathrm{o}$ persistente de APE, un tacto rectal que 
Tabla 3 Orientaciones para el diagnóstico del cáncer de próstata

\begin{tabular}{lll} 
& & $\begin{array}{c}\text { Grado de } \\
\text { recomendación }\end{array}$ \\
\hline 1 Un tacto rectal con resultado anómalo o una medición elevada de APE sérico podrían indicar \\
CP. El umbral exacto de lo que se consideraría un valor normal de APE no se ha determinado, \\
pero a menudo se utilizan valores aproximados $<2-3 \mathrm{ng} / \mathrm{ml}$ para varones jóvenes \\
El diagnóstico del CP depende de la confirmación histopatológica (o citológica) \\
Sólo estarían indicadas biopsia e investigaciones de estadificación adicionales si repercuten en \\
el tratamiento del paciente \\
La biopsia sistémica de tipo BTE es el método recomendado en la mayoría de casos en que se \\
sospeche la presencia de CP. Se recomienda un mínimo de 10 muestras obtenidas mediante \\
biopsia sistemática dirigida lateralmente, con quizás más muestras en próstatas con un \\
volumen > 40 ml \\
No se recomiendan las biopsias de la zona de transición en el primer conjunto de biopsias \\
debido a las bajas tasas de detección. \\
Se justificaría un conjunto de biopsias de repetición en casos con indicación persistente (tacto \\
rectal con resultado anómalo, APE elevado o hallazgos histopatológicos que sugieran tumor \\
maligno en la biopsia inicial) en cuanto a biopsia de la próstata \\
No es posible hacer recomendaciones generales para más conjuntos de biopsias (tres o más); \\
la decisión debe tomarse en función de cada paciente individual \\
Se puede ofrecer a los pacientes inyección periprostática transrectal con anestésico local \\
como analgesia eficaz cuando se sometan a biopsias de próstata
\end{tabular}

APE: antígeno prostático específico; BTE: biopsia transrectal ecodirigida; CP: cáncer de próstata.

haga sospechar de la presencia de cáncer y una proliferación de acinos pequeños y atípicos en la próstata. Los intervalos óptimos siguen sin estar del todo claros. Cuanto más tarde se repita la biopsia mayor será la tasa de detección ${ }^{29}$. La neoplasia intraepitelial prostática (NIP) de alto grado sólo sería indicación para una nueva biopsia si se produjese en varios focos ${ }^{29}$ (nivel de evidencia: $2 a$ ). Si sigue existiendo sospecha clínica de CP, a pesar de biopsias de próstata con resultado negativo, puede utilizarse la resonancia magnética (RM) para investigar la posibilidad de un CP situado en la zona anterior, seguida de biopsias de tipo BTE o guiadas por RM en la zona sospechosa.

El diagnóstico del CP se basa en el examen histológico ${ }^{30}$. Deben considerarse técnicas de tinción auxiliares (por ejemplo tinción de células basales) y secciones adicionales (más profundas) si se identifica una lesión glandular donde se sospeche la presencia de cáncer ${ }^{30}$.

Debe indicarse la proporción de biopsias con resultado positivo para carcinoma y el grado de Gleason mediante el sistema adoptado en $2005^{31}$ por cada biopsia. No debe realizarse diagnóstico con un grado de Gleason $\leq 4$ en biopsias de próstata ${ }^{31}$. Debe registrarse la proporción (porcentaje) o longitud (en milímetros) de la afectación tumoral por biopsia ${ }^{30,31}$ y extensión extraprostática, si existiese. Suele registrarse la presencia de NIP de alto grado y de invasión perineural.

Es necesario indicar claramente la presencia y extensión de cualquier foco de adenocarcinoma localizado en las biopsias realizadas (por ejemplo $<1 \mathrm{~mm} 0<1 \%$ ), ya que podría ser evidencia suficiente como para realizar más pruebas diagnósticas sobre la muestra o para volver a realizar una biopsia antes de seleccionar la terapia ${ }^{32,33}$.

La decisión de continuar con más pruebas diagnósticas o de estadificación depende de las opciones de tratamiento que estén disponibles para el paciente, teniendo en cuenta las preferencias, edad y comorbilidad del paciente ${ }^{34-41}$. Por lo general, pueden evitarse los procedimientos que no sirvan para decidir sobre el tratamiento. La tabla 3 y la tabla 4 resumen las orientaciones para el diagnóstico y la estadificación.

\section{Tratamiento local primario del cáncer de próstata}

El tratamiento terapéutico del CP, incluso en una enfermedad clínicamente localizada, ha aumentado cada vez más su complejidad debido a las diversas opciones terapéuticas disponibles específicas de cada estadio. Es recomendable: a) aconsejar a los pacientes con CP de bajo riesgo (APE < 10 $\mathrm{ng} / \mathrm{ml}$, grado de Gleason 6 en biopsia y cT1c-cT2a) o CP de riesgo intermedio (APE de 10,1-20 ng/ml, grado de Gleason 7 en biopsia o cT2b-c) en un entorno interdisciplinar con un urólogo y un radio-oncólogo; $b$ ) debatir opciones de tratamiento neoadyuvante y adyuvante en pacientes con $C P$ de alto riesgo (APE $<20 \mathrm{ng} / \mathrm{ml}$, grado de Gleason 8-10 en biopsia $0 \geq$ CT3a) con miembros de una comisión multidisciplinar de evaluación de tumores; y c) documentar detalladamente qué orientaciones se utilizaron en el proceso de toma de decisiones si no fue posible utilizar un enfoque multidisciplinar.

Normalmente es imposible afirmar si una terapia es claramente superior a otra, debido a la ausencia de ensayos aleatorios controlados en este campo. Sin embargo, tomando como referencia la literatura disponible, es posible realizar algunas recomendaciones. La tabla 3 presenta un resumen, subdividido por estadio en el momento del diagnóstico, así como varias sugerencias sobre las diferentes opciones de tratamiento disponibles. 
Tabla 4 Orientaciones para la estadificación del cáncer de próstata

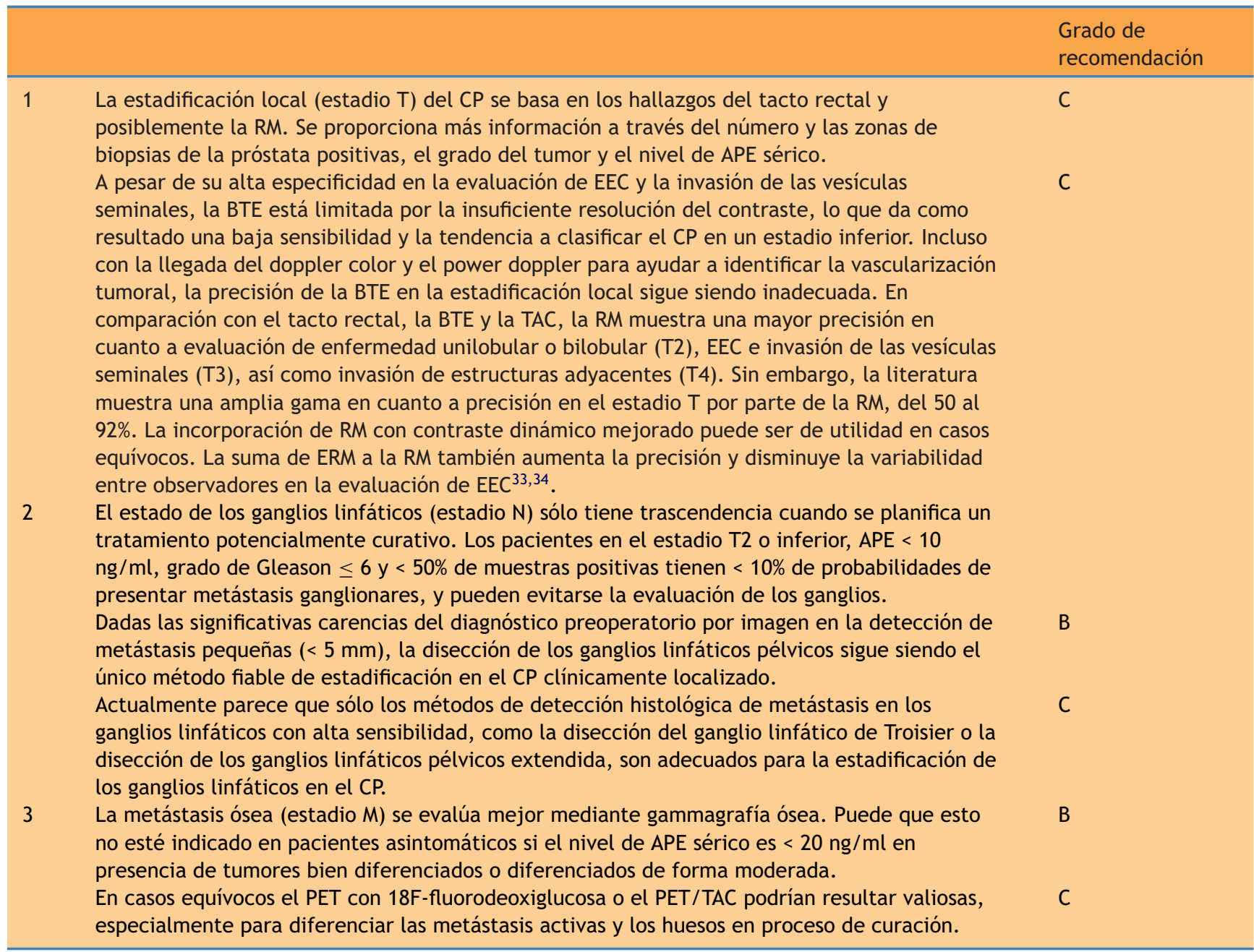

APE: antígeno prostático específico; BTE: biopsia transrectal ecodirigida; CP: cáncer de próstata; EEC: extensión extracapsular; ERM: espectroscopia por resonancia magnética; RM: resonancia magnética; TAC: tomografía axial computarizada; TEP: tomografía por emisión de positrones.

\section{Seguimiento activo}

El seguimiento activo debe diferenciarse de la espera en observación o «watchful waiting» (WW). Esta última se basa en un tratamiento no curativo sintomático diferido en pacientes que no son candidatos para una terapia local agresiva, mientras que el primero debe considerarse como terapia adecuada para aquellos a los que también se les pueda ofrecer un enfoque curativo. Los pacientes con CP de riesgo muy bajo no se someten a tratamiento inicialmente, pero sí a seguimiento y a tratamiento con intención curativa si durante el seguimiento se produce una progresión o existe una amenaza de posible progresión.

El seguimiento activo se concibió con el objetivo de reducir el cociente de tratamiento excesivo en pacientes con $\mathrm{CP}$ de bajo riesgo clínicamente limitado ${ }^{42,43}$, demostrando que los varones con CP bien diferenciado tienen una tasa de supervivencia específica del CP a 20 años del 80-90\%. Sólo están disponibles para su uso como evidencia para este enfoque los datos procedentes de ensayos clínicos aleatorios no maduros con un seguimiento activo $<10$ años.
De acuerdo con datos recientes, los varones con CP de bajo riesgo y una esperanza de vida > 10 años son buenos candidatos para el seguimiento activo, y sólo un $30 \%$ de los varones requerirá una intervención radical diferida ${ }^{44}$. Los varones con una esperanza de vida > 15 años tienen un mayor riesgo de fallecer por $C P$ (nivel de evidencia: 3).

En distintas series se han identificado varios criterios de selección de posibles pacientes para un seguimiento $\operatorname{activo}^{45}$ :

1. CP clínicamente limitado (T1-T2).

2. Grado de Gleason $\leq 6$.

3. Tres o menos biopsias que indiquen la presencia de cáncer.

4. $\leq 50 \%$ de cada biopsia que indique la presencia de cáncer. 5. $\mathrm{APE}<10 \mathrm{ng} / \mathrm{ml}$.

Además, se aplicaron diferentes criterios para definir la progresión del cáncer ${ }^{45,46}$, aunque todos los grupos se valieron de estos criterios: 
1. Tiempo de duplicación del APE con un umbral de entre $\leq 2 \mathrm{y} \leq 4$ años.

2. Progresión del grado de Gleason hasta $\geq 7$ en una nueva biopsia, en intervalos de 1 a 4 años.

3. Progresión del APE $<10 \mathrm{ng} / \mathrm{ml}$.

Sin embargo, la función del tiempo de duplicación del APE a la hora de identificar la necesidad de intervención ha cambiado recientemente ${ }^{47}$. En una cohorte de 290 varones que se sometieron a seguimiento activo de CP de bajo riesgo, el 35\% desarrolló una progresión en las biopsias (grado de Gleason $\geq 7$, más de dos muestras con resultado positivo 0 $>50 \%$ de afectación en las muestras). El tiempo de duplicación del APE no se asoció significativamente a la progresión en las biopsias $(p=0,83)$, ni tampoco la velocidad del APE $(p=0,06)$. En otro estudio, el $36 \%$ de los varones sometidos a un seguimiento activo mostró una progresión de la enfermedad en las nuevas biopsias ${ }^{48}$. La probabilidad de 5 años sin progresión fue del $82 \%$ para pacientes con una primera biopsia de repetición con resultado negativo, en comparación con el $50 \%$ de pacientes con una nueva biopsia positiva. Ambos ensayos subrayan la necesidad de nuevas biopsias de seguimiento anuales para la supervisión adecuada de los varones con un seguimiento activo, independientemente de los resultados del tiempo de duplicación del APE.

\section{Tratamiento conservador en cáncer de próstata localmente avanzado}

La literatura que versa sobre el tratamiento diferido del CP localmente avanzado es escasa. En un reciente ensayo clínico aleatorio prospectivo de fase 3 (EORTC 30981), se procedió a realizar una distribución aleatoria de 985 pacientes con CP T0-4 N0-2 MO no admitidos en un tratamiento local con intención curativa entre terapia de bloqueo hormonal o de privación androgénica (TPA) inmediata, o TPA sólo en casos de progresión sintomática de la enfermedad o de aparición de complicaciones graves ${ }^{49}$. Tras un seguimiento medio de 7,8 años la TPA inmediata dio como resultado un aumento modesto, pero con estadísticamente significativo, de la tasa de supervivencia general, pero no se generaron diferencias significativas en cuanto a mortalidad por CP o supervivencia sin síntomas. El tiempo transcurrido desde la aleatorización hasta la progresión de la enfermedad resistente a las hormonas no presentó diferencias significativas. La media de tiempo transcurrido desde la inclusión en el estudio hasta el inicio del tratamiento diferido fue de 7 años. En este grupo, 126 pacientes $(25,6 \%)$ fallecieron sin recibir tratamiento (el $44 \%$ de los fallecimientos en este grupo de estudio). Es más, los autores identificaron factores de riesgo significativos asociados a un resultado significativamente peor ${ }^{50}$. En ambos grupos de estudio los pacientes con un APE inicial > $50 \mathrm{ng} / \mathrm{ml}$ presentaron un riesgo $>3,5$ veces superior de fallecer por CP que los pacientes con un APE inicial $\leq 8 \mathrm{ng} / \mathrm{ml}$. Si el APE inicial se encontraba entre $8 \mathrm{ng} / \mathrm{ml}$ y $50 \mathrm{ng} / \mathrm{ml}$, el riesgo de fallecimiento por cáncer era aproximadamente 7,5 veces superior en pacientes con un tiempo de duplicación del APE $<12$ meses que en pacientes con un tiempo de duplicación del APE $>12$ meses. El tiempo transcurrido hasta una recaída del APE tras respuesta a la TPA inmediata presentaba una relación significativa con el APE inicial, lo cual apunta a que el APE inicial también podría reflejar la agresividad de la enfermedad.

\section{Prostatectomía radical}

La PR es el único tratamiento para el CP localizado que ha mostrado ventajas en cuanto a la supervivencia específica del cáncer al compararlo con la WW en un ensayo aleatorio prospectivo ${ }^{51,52}$. La mayoría de los pacientes admitidos presentaba riesgo intermedio, y no padecía de CP detectado mediante cribado, de modo que estos datos no se pueden transferir automáticamente a la práctica rutinaria diaria. La PR con conservación nerviosa representa la técnica de elección en todos los varones con una función eréctil normal y enfermedad limitada al órgano. La necesidad y extensión de la linfadenectomía pélvica son motivo de disputa. El riesgo de afectación ganglionar es bajo en varones con CP de bajo riesgo y $<50 \%$ de muestras con resultado positivo ${ }^{53}$. En varones con $\mathrm{CP}$ de riesgo intermedio y alto siempre debería realizarse una linfadenectomía pélvica extendida ${ }^{54}$.

El tratamiento del CP CT3 debe presentar ante todo un enfoque multimodal debido a la alta probabilidad de ganglios linfáticos positivos y/o márgenes de resección positivos $^{55-60}$. La estadificación excesiva del CP cT3 es relativamente frecuente y se produce en el $13-27 \%$ de los $\operatorname{casos}^{55,56}$. El problema sigue siendo la selección de pacientes antes de la intervención quirúrgica que no tengan ni afectación ganglionar ni invasión de las vesículas seminales. Los nomogramas que incluyan el nivel de APE, estadio y grado de Gleason pueden ser de utilidad a la hora de predecir el estadio patológico de la enfermedad ${ }^{58}$. La PR para el cáncer T3 requiere de la suficiente experiencia quirúrgica para mantener un nivel aceptable de morbilidad y mejorar los resultados oncológicos, con excelentes tasas de supervivencia específicas de cáncer del 95,90 y 79\% a los 5,10 y 15 años, respectivamente $e^{59,60}$.

El bloqueo hormonal neoadyuvante no ofrece una ventaja significativa en cuanto a tasa de supervivencia general y supervivencia sin progresión, por lo que no tiene ninguna función en cuanto a tratamiento quirúrgico del $C P^{61}$.

La TPA adyuvante tras la PR siempre ha sido objeto de debate ${ }^{62}$. Aunque el único ensayo aleatorio prospectivo mostró ventajas significativas en la tasa de supervivencia para la TPA inmediata en casos de la enfermedad positiva en ganglios ${ }^{63}$, es preciso reconocer que la mayoría de los pacientes tenía una adenopatía y que el 70\% también presentaba márgenes positivos $\mathrm{y} / \mathrm{o}$ invasión de las vesículas seminales. No se sabe si la TPA adyuvante en pacientes con afectación mínima de los ganglios daría los mismos resultados positivos. La actualización más reciente del Ensayo para el diagnóstico precoz del cáncer de próstata (Early Prostate Cancer [EPC]) no ha mostrado ninguna ventaja en la tasa de supervivencia general al incorporar $150 \mathrm{mg}$ de bicalutamida/día al tratamiento estándar ${ }^{64}$. En un reciente análisis retrospectivo de la base de datos Surveillance, Epidemiology, and End Results se detectaron ganglios linfáticos positivos en 731 varones sometidos a PR entre 1991 y 1999; de ellos, 209 recibieron TPA adyuvante ${ }^{65}$. No se encontraron diferencias estadísticamente significativas en cuanto a tasa de supervivencia general entre el grupo de TPA adyuvante y el grupo sin TPA, y tampoco diferencias estadísticamente 
significativas en la supervivencia con TPA adyuvante a los $90,150,180$ y 365 días.

No se pueden hacer recomendaciones finales para los pacientes con afectación microscópica exclusivamente de los ganglios linfáticos.

\section{Radioterapia externa adyuvante para el cáncer de próstata pT3 o pTxR1}

Tres ensayos aleatorios prospectivos han evaluado la función de la radioterapia postoperatoria inmediata. Aunque los criterios de inclusión fueron diferentes, todos los ensayos llegaron a la conclusión de que la radioterapia postoperatoria inmediata mejora significativamente la supervivencia clínica o biológica a los 5 años aproximadamente en un $20 \%$ $(p<0,0001)^{66-68}$. La radioterapia postoperatoria inmediata demostró que se toleraba bien con un riesgo de toxicidad urinaria de grado 3-4 en proporciones $\leq 3,5 \%$.

Los resultados actualizados del ensayo 8794 del Southwest Oncology Group ${ }^{69}$ con un seguimiento medio de 11,5 años mostraron que la radiación adyuvante mejoró significativamente la supervivencia sin metástasis a 15 años (46 frente a $38 \% ; p=0,036$ ) y la tasa de supervivencia general (47 frente a $37 \%$; $p=0,053$ ) en comparación con la radioterapia diferida. La tabla 2 resume las orientaciones para la PR.

De este modo, a los pacientes clasificados como T1-2 N0 (o T3 N0 con factores de pronóstico seleccionados), pT3 pNO con alto riesgo de fallo local tras una PR por márgenes positivos y/o invasión de vesículas seminales y APE negativa se les puede ofrecer dos opciones dentro de un consentimiento informado: a) bien radioterapia inmediata de 60-64 Gy en el lecho quirúrgico ${ }^{66-68}$ tras la recuperación de la función urinaria; $b$ ) bien supervisión clínica y biológica seguida de radioterapia de rescate de 66 Gy como mínimo en el mejor de los casos, si el APE aumenta pero no supera los $0,5 \mathrm{ng} / \mathrm{ml}$.

\section{Radioterapia}

La radioterapia conformada en tres dimensiones (RTC3D) es la norma, y la radioterapia de intensidad modulada (RIM), una forma optimizada de la RTC3D, se está utilizando cada vez más como radioterapia guiada por imágenes. La tabla 2 resume las orientaciones para radioterapia en el CP.

Para radioterapia externa se recomienda una dosis mínima de 74 Gy para el tratamiento del CP de bajo riesgo, debido a que la supervivencia bioquímica sin la enfermedad es significativamente superior cuando se compara con una dosis < 72 Gy (69 frente a 63\%; $p=0,046)^{70}$.

Para el CP de riesgo intermedio muchas series han mostrado un impacto significativo del aumento de la dosis en cuanto a supervivencia sin evolución a 5 años en el CP cT1c$\mathrm{T} 3$, con una dosis entre 76 y $81 \mathrm{~Gy}^{71}$.

En pacientes con enfermedad de alto riesgo una irradiación externa con aumento de la dosis mejora la supervivencia bioquímica sin enfermedad a 5 años ${ }^{72}$, pero parece insuficiente para cubrir el riesgo de recaída sistémica. Para el CP de riesgo intermedio y muy localizado una combinación de irradiación externa con 6 meses de TPA dio como resultado una mejora del $13 \%$ en la tasa de supervivencia general a 8 años $(p<0,001)^{72,73}$. Para el CP localmente avanzado los datos del ensayo EORTC-22961 mostraron una ventaja del $4,7 \%$ en tasa de supervivencia general tras un seguimiento medio de 5,2 años a favor de la TPA de tres años, al compararla con la TPA a corto plazo ${ }^{73}$.

Por lo tanto, la TPA concomitante (con o sin neoadyuvante) y adyuvante durante tres años se haría obligatoria, y representa la norma actual para el tratamiento radioterapéutico del CP de alto riesgo.

Diversos ensayos aleatorios prospectivos han evaluado la eficacia oncológica de la TPA con o sin radioterapia externa $(\mathrm{RTE})^{74-76}$. Los ensayos del Scandinavian Prostate Cancer Group-7 agruparon a 875 varones con CP localmente avanzado asignados aleatoriamente a tratamiento endocrino o a TPA con RTE con una dosis de $70 \mathrm{~Gy}$ como mínimo ${ }^{74}$. Tras un seguimiento medio de 7,6 años, la mortalidad específica del cáncer fue significativamente superior en el grupo de estudio de TPA (23,9 frente al 11,9\%); ese fue también el caso de la mortalidad general $(39,4$ frente al $29,6 \%)$ y la tasa de fracaso del APE $(74,7$ frente al 25,5\%; $p<0,0001)$. Recientemente se han presentado dos ensayos clínicos aleatorios prospectivos relativos al mismo asunto en forma de resumen ${ }^{75,76}$. Un estudio canadiense asignó aleatoriamente a 1.205 varones con CP localmente avanzado a tratamiento con TPA o TPA con RTE de $65-69 \mathrm{~Gy}^{75}$. Tras un seguimiento medio de 6 años la adición de la RTE redujo significativamente el riesgo de fallecimiento (cociente de riesgos instantáneos: 0,77; $p=0,033$ ) con una tasa acumulada de fallecimientos específicos por la enfermedad a 10 años del 15 frente al 23\%. Un estudio francés asignó aleatoriamente a 263 pacientes con CP localmente avanzado a tratamiento con TPA o TPA y $\mathrm{RTE}^{76}$. Con un seguimiento mínimo de 5 años el tratamiento combinado logró resultados significativamente mejores en lo relativo a supervivencia sin progresión $(60,9$ frente al $8,5 \% ; p=0,001)$, progresión locorregional $(9,7$ frente al $29 \%$; $\mathrm{p}=0,0002$ ) y progresión metastática (3 frente al 10,8\%; $p=0,018)$.

\section{Braquiterapia transperineal de baja dosis}

La braquiterapia transperineal es una técnica segura y eficaz para el CP de bajo riesgo. Existe un consenso acerca de los siguientes criterios de selección ${ }^{77}$ :

1. Estadio cT1c-T2a NO, MO.

2. Grado de Gleason $\leq 6$ evaluado sobre un número suficiente de biopsias aleatorias.

3. Nivel inicial de APE $\leq 10 \mathrm{ng} / \mathrm{ml}$.

4. $\leq 50 \%$ de muestras que indiquen la presencia de cáncer.

5. Volumen prostático $<50 \mathrm{ml}$.

6. Un buen baremo internacional de síntomas prostáticos.

Algunas instituciones han publicado resultados sobre implantes permanentes con un seguimiento medio de entre 36 y 120 meses $^{78,79}$. Se estableció una supervivencia sin recaídas a los 5 y 10 años entre el 71 y el 93\%, y entre el 65 y el $85 \%$, respectivamente. No se observan ventajas al añadir TPA neoadyuvante o adyuvante a braquiterapia de baja dosis ${ }^{37}$. 


\section{Irradiación de los ganglios linfáticos pélvicos}

En lo relativo a las posibles ventajas de la irradiación de los ganglios linfáticos pélvicos en varones con CP localizado de alto riesgo, el ensayo de Groupe d'Etude des Tumeurs UroGénitales asignó aleatoriamente a 444 pacientes para RTE en próstata (66-70 Gy) o en lecho prostático y en ganglios linfáticos pélvicos $(46 \mathrm{~Gy})^{79}$. La tasa de supervivencia general y la supervivencia sin progresión a 5 años fueron similares en ambos grupos de estudio, por lo que no existiría una indicación general para la irradiación de los ganglios linfáticos pélvicos.

\section{Técnicas innovadoras}

La RIM permite a los radio-oncólogos aumentar las dosis de radiación de forma homogénea hasta un máximo de 86 Gy dentro del volumen objetivo, al tiempo que se respetan las dosis de tolerancia en órganos con riesgo.

El Memorial Sloan-Kettering Cancer Centre es la institución con más amplia experiencia en esta técnica, y han actualizado recientemente sus resultados, aportando información sobre control de la enfermedad y toxicidad en dos cohortes de pacientes ${ }^{80,81}$.

En la primera cohorte se trató a 561 pacientes con enfermedad limitada al órgano con una dosis de $81 \mathrm{~Gy}$. Las tasas de supervivencia sin recaídas actuariales del APE en 8 años para pacientes en grupos de riesgo favorable, intermedio y no favorable fueron del 85,76 y $72 \%$, respectivamente, de acuerdo con la definición de la American Society for Radiation Oncology vigente en ese momento.

En la segunda cohorte se aplicó tratamiento a 478 pacientes con enfermedad limitada al órgano con una dosis de $86,4 \mathrm{~Gy}$. Las tasas de supervivencia sin recaídas actuariales del APE a 5 años según el nadir más $2 \mathrm{ng} / \mathrm{ml}$, fueron del 98,85 y $70 \%$ para grupos de riesgo bajo, intermedio y alto, respectivamente.

\section{Radioterapia con protones e iones de carbono}

En teoría los haces de protones son una alternativa atractiva a la radioterapia con fotones para el CP, ya que depositan casi toda la dosis de radiación al final del recorrido de la partícula en el tejido (pico de Bragg), al contrario que los fotones, que depositan la radiación a todo lo largo de su trayectoria. Además, el gradiente de radiación de los haces de protones más allá de su profundidad de deposición es muy marcado, lo que significa que los tejidos normales críticos que se encuentren más allá de esa profundidad pueden dejarse intactos de manera eficaz. Por el contrario, los haces de fotones siguen depositando energía hasta abandonar el cuerpo, incluida una dosis de salida.

Sólo existe un ensayo aleatorio que haya incorporado radioterapia con protones en un grupo de estudio y que ofrezca información sobre resultados a largo plazo ${ }^{82,83}$. El ensayo 9509 del Proton Radiation Oncology Group asignó aleatoriamente a 393 varones con CP clínicamente localizado a RTE de 70,2Gy frente a 79,2Gy de radiación combinada de fotones y protones. Tras un seguimiento medio de 9,4 años la tasa de progresión bioquímica estimada a 10 años para pacientes receptores de la dosis estándar fue del
$32 \%$, en comparación con el $17 \%$ de pacientes a los que se aplicó la dosis alta $(p<0,001)$. No se presentaron diferencias significativas entre ambos grupos respecto a los índices de síntomas de CP en lo relativo a la obstrucción/irritación urinaria $(23,3$ frente a 24,$6 ; p=0,36)$, incontinencia urinaria $(10,6$ frente a 9,$7 ; p=0,99)$, problemas intestinales $(7,7$ frente a 7,$9 ; p=0,70)$ y disfunción sexual $(68,2$ frente a 65,9 ; $p=0,65$ ). Sin embargo, se necesitaría un ensayo aleatorio prospectivo en el que se empleasen dosis equivalentes de RIM y radiación con fotones para evaluar la eficacia oncológica de los fotones.

\section{Seguimiento de los pacientes con cáncer de próstata}

Los pacientes a los que se diagnostica $\mathrm{CP}$, sometidos a tratamiento local con intención curativa, suelen ser sometidos a un seguimiento de 10 años al menos, o hasta una edad tan avanzada que convierta el seguimiento en superfluo (tabla 5). La determinación del APE sérico, junto con una anamnesis específica de la enfermedad, pueden complementarse con un tacto rectal si se sospecha enfermedad localmente recurrente.

\section{Opciones alternativas de tratamiento local del cáncer de próstata}

Además de la PR, la RTE y/o la braquiterapia, la ablación crioquirúrgica de la próstata y el ultrasonido focalizado de alta intensidad han surgido como opciones terapéuticas alternativas en pacientes con CP clínicamente localizado no aptos para la $\mathrm{PR}^{84,85}$. Sin embargo, en el momento de elaborar este documento, los datos respecto a ultrasonido focalizado de alta intensidad no eran lo suficientemente amplios como para tenerlos en cuenta en recomendaciones de tratamientos. Al aplicar el enfoque del Grading of Recommendations Assessment, Development and Evaluation, los datos disponibles en cuanto a eficacia y seguridad del ultrasonido focalizado de alta intensidad en el CP son de muy baja calidad, sobre todo porque el diseño del estudio no incluye grupos de control. Es necesario abundar en la investigación para examinar el uso del ultrasonido focalizado de alta intensidad en el CP. Los siguientes factores pueden ser indicaciones: CP de riesgo bajo o intermedio y tamaño de la próstata $<40 \mathrm{ml}$ en el momento de la terapia.

Existe una carencia de resultados a largo plazo, y las tasas de supervivencia sin progresión bioquímica a 5 años son inferiores a las de la PR en pacientes de bajo riesgo, por lo que debe informarse a los pacientes en consecuencia. Se han publicado recientemente los resultados de un ensayo aleatorio sobre RTE frente a ablación crioquirúrgica de la próstata en pacientes con CP clínicamente localizado, ensayo que presentaba resultados prometedores ${ }^{86}$. Se asignaron 244 varones con CP de riesgo bajo e intermedio a ambos grupos de estudio del tratamiento, siendo todos los pacientes destinatarios de TPA adyuvante. Tras un seguimiento medio de 100 meses no se observaron diferencias en cuanto a progresión de la enfermedad a 36 meses, tasa de supervivencia general y supervivencia específica de la enfermedad. Los resultados positivos pueden deberse al hecho de que se proporcionó TPA neoadyuvante, y a que ambos 
Tabla 5 Orientaciones para el seguimiento tras un tratamiento primario con intención curativa

\begin{tabular}{|c|c|}
\hline & $\begin{array}{l}\text { Grado de } \\
\text { recomendación }\end{array}$ \\
\hline $\begin{array}{l}\text { En pacientes asintomáticos, las pruebas recomendadas para un seguimiento rutinario son una } \\
\text { anamnesis específica de la enfermedad y una medición de APE sérico, complementadas con } \\
\text { un tacto rectal. Estas pruebas deberían realizarse a los } 3,6 \text { y } 12 \text { meses del tratamiento; a } \\
\text { continuación, cada } 6 \text { meses durante } 3 \text { años y, por último, anualmente. }\end{array}$ & B \\
\hline $\begin{array}{l}\text { Tras una PR un nivel de APE sérico }>0,2 \mathrm{ng} / \mathrm{ml} \text { puede asociarse a una enfermedad residual o } \\
\text { recurrente. }\end{array}$ & B \\
\hline $\begin{array}{l}\text { Tras una radioterapia un nivel de APE }>2 \mathrm{ng} / \mathrm{ml} \text { en aumento por encima del nadir del APE en } \\
\text { lugar de un valor de umbral específico es la señal más fiable de una enfermedad persistente } \\
\text { o recurrente. }\end{array}$ & B \\
\hline $\begin{array}{l}\text { Tanto un ganglio palpable como un nivel de APE sérico en aumento pueden ser señales de } \\
\text { recurrencia local de una enfermedad. }\end{array}$ & B \\
\hline $\begin{array}{l}\text { La detección de una recurrencia local mediante una BTE y una biopsia sólo se recomienda si } \\
\text { afecta al plan de tratamiento. En la mayoría de los casos la BTE y la biopsia no son } \\
\text { necesarias antes de una terapia de segunda línea. }\end{array}$ & B \\
\hline $\begin{array}{l}\text { Una metástasis puede detectarse mediante una TAC/RM pélvica o una gammagrafía ósea. En } \\
\text { pacientes asintomáticos estos exámenes pueden omitirse si el nivel de APE sérico es }<20 \\
\mathrm{ng} / \mathrm{ml} \text {, pero los datos sobre este tema son escasos. }\end{array}$ & C \\
\hline $\begin{array}{l}\text { Las gammagrafías óseas y otros estudios de diagnóstico por imagen rutinarios no se } \\
\text { recomiendan para pacientes asintomáticos. Si un paciente tiene dolor óseo, debe }\end{array}$ & $B$ \\
\hline
\end{tabular}

APE: antígeno prostático específico; BTE: biopsia transrectal ecodirigida; PR: prostatectomía radical; RM: resonancia magnética; TAC: tomografía axial computarizada.

grupos de estudio y el número de pacientes eran demasiado pequeños como para llegar a conclusiones clínicamente significativas.

\section{Versión completa}

Este texto representa un resumen; para obtener información más detallada y una lista completa de referencias remitimos a los lectores a la versión completa del texto. Esta guía de la EAU (ISBN 978-90-79754-70-0) está disponible en el sitio web de la EAU (www.uroweb.org).

\section{Contribuciones de los autores}

Axel Heidenreich dispuso de total acceso a los datos del estudio, por lo que asume la total responsabilidad respecto a la integridad de los datos y la precisión del análisis de datos.

Conceptualización y diseño del estudio: Heidenreich, Bellmunt, Bolla, Joniau, Mason, Matveev, Mottet, Schmid, van der Kwast, Wiegel, Zattoni.

Recogida de datos: Heidenreich, Bellmunt, Bolla, Joniau, Mason, Matveev, Mottet, Schmid, Van der Kwast, Wiegel, Zattoni.

Análisis e interpretación de datos: Heidenreich, Bellmunt, Bolla, Joniau, Mason, Matveev, Mottet, Schmid, Van der Kwast, Wiegel, Zattoni.

Borrador del manuscrito: Heidenreich.

Revisión crítica del manuscrito en cuanto a contenido intelectual de entidad: Heidenreich, Bellmunt, Bolla, Joniau, Mason, Matveev, Mottet, Schmid, van der Kwast, Wiegel, Zattoni.

Análisis estadístico: no realizado.
Obtención de financiación: no realizada.

Asistencia administrativa, técnica o material: Heidenreich, Bellmunt, Bolla, Joniau, Mason, Matveev, Mottet, Schmid, Van der Kwast, Wiegel, Zattoni.

Supervisión: ninguna.

Otros aspectos (especificar): ninguno.

\section{Fuentes de financiación}

Declaro por el presente documento que los conflictos de intereses, incluyendo intereses específicos de financiación y relaciones o vínculos de relevancia en relación con el tema o los materiales tratados en el manuscrito (por ejemplo empresa contratante/vínculos, becas o financiación, asesoramiento, honorarios, propiedad de acciones u opciones, testimonio experto, derechos o patentes presentadas, otorgadas o pendientes) son los siguientes: ninguno.

Financiación/apoyos y papel del patrocinador: ninguno.

\section{Bibliografía}

1. Heidenreich A, Aus G, Bolla M, Joniau S, Matveev VB, Schmid HP, et al. EAU guidelines on prostate cancer. Eur Urol. 2008;53:68-80.

2. US Department of Health and Human Services. Agency for Healthcare Research and Quality Web site. Disponible en: http://www. ahcpr.gov/.

3. Jemal A, Siegel R, Ward E, Hao Y, Xu J, Murray T, et al. Cancer statistics 2008. CA Cancer J Clin. 2008;58:71-96.

4. Ferlay J, Parkin DM, Steliarova-Foucher E. Estimates of cancer incidence and mortality in Europe 2008. Eur J Cancer. 2010;46:765-81. 
5. Bratt O. Hereditary prostate cancer: clinical aspects. J Urol. 2002;168:906-13.

6. TNM classification of malignant tumors. En: Sobin LH, Gospodariwicz M, Wittekind C, editores. UICC International Union Against Cancer. 7. $\stackrel{a}{a}$ ed. Hoboken, NJ: Wiley-Blackwell; 2009. p. 243-8.

7. Gleason DF, Mellinger GT. Prediction of prognosis for prostatic adenocarcinoma by combined histological grading and clinical staging. J Urol. 1974;111:58-64.

8. Ilic D, O'Connor D, Green S, Wilt T. Screening for prostate cancer: a Cochrane systematic review. Cancer Causes Control. 2007; 18:279-85.

9. Andriole GL, Crawford ED, Grubb III RL, Buys SS, Chia D, Church TR, et al. Mortality results from a randomized prostate-cancer screening trial. N Engl J Med. 2009;360:1310-9.

10. Schröder FH, Hugosson J, Roobol MJ, Tammela TL, Ciatto S, Nelen $\mathrm{V}$, et al. Screening and prostate-cancer mortality in a randomized European study. N Engl J Med. 2009;360:1320-8.

11. Van Leeuwen PJ, Connolly D, Gavin A, Roobol MJ, Black A, Bangma $\mathrm{CH}$, et al. Prostate cancer mortality in screen and clinically detected prostate cancer: estimating the screening benefit. Eur J Cancer. 2010;46:377-83.

12. Börgermann C, Loertzer $H$, Hammerer P, Fornara P, Graefen $M$, Rübben $\mathrm{H}$. Problems, objective, and substance of early detection of prostate cancer [en alemán]. Urologe A. 2010;49:181-9.

13. Carter HB, Kettermann AE, Ferrucci L, Landis P, Trock BJ, Metter EJ. Prostate specific antigen testing among the elderly; when to stop? J Urol. 2008;174 Suppl 1:600.

14. Carvalhal GF, Smith DS, Mager DE, Ramos C, Catalona WJ. Digital rectal examination for detecting prostate cancer at prostate specific antigen levels of $4 \mathrm{ng} / \mathrm{ml}$ or less. J Urol. 1999;161:835-9.

15. Loeb S, Catalona WJ. What is the role of digital rectal examination in men undergoing serial screening of serum PSA levels? Nat Clin Pract Urol. 2009;6:68-9.

16. Stephan C, Köpke T, Semjonow A, Lein M, Deger S, Schrader M, et al. Discordant total and free prostate-specific antigen (PSA) assays: does calibration with WHO reference materials diminish the problem? Clin Chem Lab Med. 2009;47: 1325-31.

17. Thompson IM, Pauler DK, Goodman PJ, Tangen CM, Lucia MS, Parnes $\mathrm{HL}$, et al. Prevalence of prostate cancer among men with a prostate-specific antigen level $\leq 4,0 \mathrm{ng}$ per millilitre. $\mathrm{N}$ Engl J Med. 2004;350:2239-46.

18. Kobori Y, Kitagawa Y, Mizokami A, Komatsu K, Namiki M. Freeto-total prostate-specific antigen (PSA) ratio contributes to an increased rate of prostate cancer detection in a Japanese population screened using a PSA level of $2.1-10.0 \mathrm{ng} / \mathrm{ml}$ as a criterion. Int J Clin Oncol. 2008;13:229-32.

19. O'Brien MF, Cronin AM, Fearn PA, Smith B, Stasi J, Guillonneau B, et al. Pretreatment prostate-specific antigen (PSA) velocity and doubling time are associated with outcome but neither improves prediction of outcome beyond pretreatment PSA alone in patients treated with radical prostatectomy. J Clin Oncol. 2009;27:3591-7.

20. Vickers AJ, Savage C, O'Brien MF, Lilja H. Systematic review of pretreatment prostate-specific antigen velocity and doubling time as predictors for prostate cancer. J Clin Oncol. 2009;27:398-403.

21. Deras IL, Aubin SM, Blase A, Day JR, Koo S, Partin AW, et al. PCA3: a molecular urine assay for predicting prostate biopsy outcome. J Urol. 2008;179:1587-9.

22. Remzi M, Haese A, Van Poppel H, De La Taille A, Stenzl A, Hennenlotter J, et al. Follow-up of men with an elevated PCA3 score and a negative biopsy: does an elevated PCA3 score indeed predict the presence of prostate cancer? BJU Int. 2010;106:1138-42.
23. Ploussard G, Haese A, Van Poppel H, Marberger M, Stenzl A, Mulders PF, et al. The prostate cancer gene 3 (PCA3) urine test in men with previous negative biopsies: does free-tototal prostate-specific antigen ratio influence the performance of the PCA3 score in predicting positive biopsies? BJU Int. 2010;106:1143-7.

24. Hara R, Jo Y, Fujii T, Kondo N, Yokoyoma T, Miyaji Y, et al. Optimal approach for prostate cancer detection as initial biopsy: prospective randomized study comparing transperineal versus transrectal systematic 12-core biopsy. Urology. 2008;71:191-5.

25. Takenaka A, Hara R, Ishimura T, Fujii T, Jo Y, Nagai A, et al. A prospective randomized comparison of diagnostic efficiency between transperineal and transrectal 12-core prostate biopsy. Prostate Cancer Prostatic Dis. 2008;11:134-8.

26. Eichler K, Hempel S, Wilby J, Myers L, Bachmann LM, Kleijnen J. Diagnostic value of systematic biopsy methods in the investigation of prostate cancer: a systematic review. J Urol. 2006;175:1605-12.

27. Aron M, Rajeev TP, Gupta NP. Antibiotic prophylaxis for transrectal needle biopsy of the prostate: a randomized controlled study. BJU Int. 2000;85:682-5.

28. Adamakis I, Mitropoulos D, Haritopoulos K, Alamanis C, Stravodimos K, Giannopoulos A. Pain during transrectal ultrasonography guided prostate biopsy: a randomized prospective trial comparing periprostatic infiltration with lidocaine with the intrarectal instillation of lidocaine-prilocain cream. World J Urol. 2004;22:281-4.

29. Merrimen JL, Jones G, Walker D, Leung CS, Kapusta LR, Srigley JR. Multifocal high grade prostatic intraepithelial neoplasia is a significant risk factor for prostatic adenocarcinoma. J Urol. 2009;182:485-90.

30. Van der Kwast TH, Lopes C, Santonja C, et al. Guidelines for processing and reporting of prostatic needle biopsies. J Clin Pathol. 2003;56:336-40.

31. Epstein JI, Allsbrook Jr WC, Amin MB, Egevad LL, ISUP Grading Committee. The 2005 International Society of Urologic Pathology (ISUP) Consensus Conference on Gleason Grading of Prostatic Carcinoma. Am J Surg Pathol. 2005;29:1228-42.

32. Herkommer K, Kuefer R, Gschwend JE, Hautmann RE, Volkmer BG. Pathological T0 prostate cancer without neoadjuvant therapy: clinical presentation and follow-up. Eur Urol. 2004;45:36-41.

33. Trpkov K, Gao Y, Hay R, Yimaz A. No residual cancer on radical prostatectomy after positive 10-core biopsy: incidence, biopsy findings, and DNA specimen identity analysis. Arch Pathol Lab Med. 2006;130:811-6.

34. Fuchsjager M, Shukla-Dave A, Akin Barentsz O, Hricak H. Prostate cancer imaging. Acta Radiol. 2008;49:107-20.

35. Wang L, Hricak H, Kattan MW, Chen HN, Kuroiwa K, Eisemberg HF. Prediction of seminal vesicle invasion in prostate cancer: incremental value of adding endorectal MRI to the Kattan Nomogram. Radiology. 2007;242:182-8.

36. Hoivels AM, Heesakkers RAM, Adang EM, Jager GJ, Strum S, Hoogeveen $\mathrm{YL}$, et al. The diagnostic accuracy of CT and MRI in the staging of pelvic lymph nodes in patients with prostate cancer: a meta-analysis. Clin Radiol. 2008;63:387-95.

37. Husarik DB, Miralbell R, Dubs M, John H, Giger OT, Gelet A, et al. Evaluation of [(18)F]-choline PET/CT for staging and restaging of prostate cancer. Eur J Nucl Med Mol Imaging. 2008;35:253-63.

38. Schiavina R, Scattoni V, Castellucci P, Picchio M, Corti B, Briganti $A$, et al. 11C-choline positron emission tomography/computerized tomography for preoperative lymphnode staging in intermediate-risk and high-risk prostate cancer: comparison with clinical staging nomograms. Eur Urol. 2008;54:392-401.

39. Heidenreich A, Varga Z, Von Knobloch R. Extended pelvic lymphadenectomy in patients undergoing radical prostatec- 
tomy: high incidence of lymph node metastasis. J Urol. 2002;167:1681-6.

40. Briganti A, Passoni N, Ferrari M, Capitanio U, Suardi N, Gallina A, et al. When to perform bone scan in patients with newly diagnosed prostate cancer: external validation of the currently available guidelines and proposal of a novel risk stratification tool. Eur Urol. 2010;57:551-8.

41. Beheshti M, Vali R, Langsteger W. [18F]Fluorocholine PET/CT in the assessment of bone metastases in prostate cancer. Eur $\mathrm{J}$ Nucl Med Mol Imaging. 2007;34:1316-7.

42. Chodak GW, Thisted RA, Gerber GS, Johansson JE, Adolfsson J, Jones GW, et al. Results of conservative management of clinically localized prostate cancer. N Engl J Med. 1994;330: 242-8.

43. Albertsen PC, Hanley JA, Gleason DF, Barry MJ. Competing risk analysis of men aged 55 to 74 years at diagnosis managed conservatively for clinically localized prostate cancer. JAMA. 1998;280:975-80.

44. Klotz L, Zhang L, Lam A, Nam R, Mamedov A, Loblaw A. Clinical results of long-term follow-up of a large, active surveillance cohort with localized prostate cancer. J Clin Oncol. 2010;28:126-31.

45. Klotz L. Active surveillance for prostate cancer: a review. Curr Urol Rep. 2010;11:165-71.

46. Krakowsky Y, Loblaw A, Klotz L. Prostate cancer death of men treated with initial active surveillance: clinical and biochemical characteristics. J Urol. 2010;184:131-5.

47. Al Otaibi M, Ross P, Fahmy N, Jeyaganth S, Trottier H, Sircar K, et al. Role of repeated biopsy of the prostate in predicting disease progression in patients with prostate cancer on active surveillance. Cancer. 2008;113:286-92.

48. Ross AE, Loeb S, Landis P, Partin AW, Epstein JI, Kettermann $A$, et al. Prostate-specific antigen kinetics during follow-up are an unreliable trigger for intervention in a prostate cancer surveillance program. J Clin Oncol. 2010;28:2810-6.

49. Studer UE, Whelan P, Albrecht W, Casselman J, De Reijke T, Hauri $D$, et al. Immediate or deferred androgen deprivation for patients with prostate cancer not suitable for local treatment with curative intent: European Organisation for Research and Treatment of Cancer (EORTC) Trial 30891. J Clin Oncol. 2006;24:1868-76.

50. Studer UE, Collette L, Whelan P, et al. Using PSA to guide timing of androgen deprivation in patients with TO-4 N0-2 MO prostate cancer not suitable for local curative treatment (EORTC 30891). Eur Urol. 2008;53:941-9.

51. Bill-Axelson A, Holmberg L, Ruutu M, et al. Radical prostatectomy versus watchful waiting in early prostate cancer. $\mathrm{N}$ Engl J Med. 2005;352:1977-84.

52. Bill-Axelson A, Holmberg L, Filén F, Ruutu M, Garmo H, Busch C, et al. Radical prostatectomy versus watchful waiting in localized prostate cancer: the Scandinavian prostate cancer group-4 randomized trial. J Natl Cancer Inst. 2008;100:1144-54.

53. Heidenreich A, Pfister D, Thüer D, Brehmer B. Percentage of positive biopsies predicts lymph node involvement in men with low-risk prostate cancer undergoing radical prostatectomy and extended pelvic lymphadenectomy. BJU Int. 2011;107: 220-5.

54. Briganti A, Chun FK, Salonia A, Gallina A, Farina E, Da Pozzo LF, et al. Validation of a nomogram predicting the probability of lymph node invasion based on the extent of pelvic lymphadenectomy in patients with clinically localized prostate cancer. BJU Int. 2006;98:788-93.

55. Yossepowitch O, Eggener SE, Bianco Jr FJ, et al. Radical prostatectomy for clinically localized, high risk prostate cancer: critical analysis of risk assessment methods. J Urol. 2007;178:493-9.

56. Ward JF, Slezak JM, Blute ML, Bergstralh EJ, Zincke H. Radical prostatectomy for clinically advanced (cT3) prostate cancer since the advent of prostate-specific antigen testing: 15-year outcome. BJU Int. 2005;95:751-6.

57. Pierorazio PM, Guzzo TJ, Han M, et al. Long-term survival after radical prostatectomy for men with high Gleason sum in pathologic specimen. Urology. 2010;76:715-21.

58. Joniau S, Hsu CY, Lerut E, Van Baelen A, Haustermans K, Roskams T, et al. A pretreatment table for the prediction of final histopathology after radical prostatectomy in clinical unilateral T3a prostate cancer. Eur Urol. 2007;51:388-96.

59. Van Poppel H, Joniau S. An analysis of radical prostatectomy in advanced stage and high-grade prostate cancer. Eur Urol. 2008; 53:253-9.

60. Loeb S, Smith ND, Roehl KA, Catalona WJ. Intermediate-term potency, continence, and survival outcomes of radical prostatectomy for clinically high-risk or locally advanced prostate cancer. Urology. 2007;69:1170-5.

61. Shelley MD, Kumar S, Wilt T, Staffurth J, Coles B, Mason MD. A systematic review and meta-analysis of randomised trials of neo-adjuvant hormone therapy for localised and locally advanced prostate carcinoma. Cancer Treat Rev. 2009;35: 9-17.

62. Kumar S, Shelley M, Harrison C, Coles B, Wilt TJ, Mason MD. Neo-adjuvant and adjuvant hormone therapy for localised and locally advanced prostate cancer. Cochrane Database Syst Rev. 2006:CD006019.

63. Messing EM, Manola J, Yao J, Kiernan M, Crawford D, Wilding $\mathrm{G}$, et al. Immediate versus deferred androgen deprivation treatment in patients with node-positive prostate cancer after radical prostatectomy and pelvic lymphadenectomy. Lancet Oncol. 2006;7:472-9.

64. McLeod DG, Iversen P, See WA, Morris T, Armstrong J, Wirth MP, Casodex Early Prostate Cancer Trialists' Group. Bicalutamide $150 \mathrm{mg}$ plus standard care vs standard care alone for early prostate cancer. BJU Int. 2006;97:247-54.

65. Wong YN, Freedland S, Egleston B, Hudes G, Schwartz JS, Armstrong K. Role of androgen deprivation therapy for nodepositive prostate cancer. J Clin Oncol. 2009;27:100-5.

66. Bolla M, Van Poppel H, Collette L, Van Cangh P, Vekemans K, Da Pozzo L, et al. Postoperative radiotherapy after radical prostatectomy: a randomized controlled trial (EORTC trial 22911). Lancet. 2005;366:572-8.

67. Wiegel T, Bottke D, Steiner U, et al. Phase III postoperative adjuvant radiotherapy after radical prostatectomy compared with radical prostatectomy alone in $\mathrm{pT} 3$ prostate cancer with postoperative undetectable prostate-specific antigen: ARO 9602/AUO AP 09/95. J Clin Oncol. 2009;27:2924-30.

68. Swanson GP, Thompson IM, Tangen C, et al. Update of SWOG: 8794 adjuvant radiotherapy for $\mathrm{PT} 3$ prostate cancer improves metastasis free survival. Int J Radiat Oncol Biol Phys. 2008;72:S31.

69. Thompson IM, Tangen CM, Paradelo J, Lucia MS, Miller G, Troyer D, et al. Adjuvant radiotherapy for pathological T3NOMO prostate cancer significantly reduces risk of metastases and improves survival: long-term followup of a randomized clinical trial. J Urol. 2009;181:956-62.

70. Kupelian P, Kuban D, Thames H, et al. Improved biochemical relapse-free survival with increased external radiation doses in patients with localized prostate cancer: the combined experience of nine institutions in patients treated in 1994 and 1995. Int J Radiat Oncol Biol Phys. 2005;61:415-9.

71. Peeters ST, Heemsbergen WD, Koper PCM, et al. Dose-response in radiotherapy for localized prostate cancer: results of the Dutch multicenter randomized phase III trial comparing $68 \mathrm{~Gy}$ of radiotherapy with $78 \mathrm{~Gy}$. J Clin Oncol. 2006;24: 1990-6.

72. D'Amico A, Renshaw AA, Loffredo M, Kantoff PW. Androgen suppression and radiation vs radiation alone for prostate cancer; a randomized controlled trial. JAMA. 2008;299:289-95. 
73. Bolla M, De Reijke TM, Van Tienhoven G, Van den Bergh AC, Oddens J, Poortmans PM, et al. Duration of androgen suppression in the treatment of prostate cancer. $\mathrm{N}$ Engl J Med. 2009;360:2516-27.

74. Widmark A, Klepp O, Solberg A, et al., Scandinavian Prostate Cancer Group Study 7; Swedish Association for Urological Oncology 3. Endocrine treatment, with or without radiotherapy, in locally advanced prostate cancer (SPCG-7/SFUO-3): an open randomised phase III trial. Lancet. 2009;373:301-8.

75. Warde PR, Mason MD, Sydes MR, et al. Intergroup randomized phase III study of androgen deprivation therapy (TPA) plus radiation therapy (RT) in locally advanced prostate cancer (PCa) (NCIC-CTG, SWOG, MRC-UK, INT: T94-0110; NCT00002633). J Clin Oncol. 2010;28 Suppl:18s.

76. Mottet N, Peneau M, Mazeron J, Molinie V, Richaud P. Impact of radiotherapy (RT) combined with androgen deprivation (TPA) versus TPA alone for local control in clinically locally advanced prostate cancer. J Clin Oncol. 2010;28 Suppl:15s.

77. Ash D, Flynn A, Batterman J, De Reijke T, Lavagnini P, Blank L, ESTRA/EAU Urological Brachytherapy Group; EORTC Radiotherapy Group. ESTRO/EAU/EORTC recommendations on permanent seed implantation for localized prostate cancer. Radiother Oncol. 2000;57:315-21.

78. Voulgaris S, Nobes JP, Laing RW, Langley SE. State-of-the-art: prostate LDR brachytherapy. Prostate Cancer Prostatic Dis. 2008;11:237-40.

79. Taira AV, Merrick GS, Butler WM, Galbreath RW, Lief J, Adamovich E, et al. Long-term outcome for clinically localized prostate cancer treated with permanent intersti- tial brachytherapy. Int J Radiat Oncol Biol Phys. 2011;79: 1336-42.

80. Pommier P, Chabaud S, Lagrange JL, Richaud P, Lesaunier F, Le Prise $\mathrm{E}$, et al. Is there a role for pelvic irradiation in localized prostate adenocarcinoma? Preliminary results of GETUG-01. J Clin Oncol. 2007;25:5366-73.

81. Zelefsky MJ, Chan H, Hunt M, Yamada Y, Shippy AM, Amols H. Long-term outcome of high dose intensity modulated radiation therapy for patients with clinically localized prostate cancer. J Urol. 2006;176:1415-9.

82. Cahlon O, Zelefsky MJ, Shippy A, Chan H, Fuks Z, Yamada Y, et al. Ultrahigh dose $(86.4 \mathrm{~Gy})$ IMRT for localized prostate cancer: toxicity and biochemical outcomes. Int J Radiat Oncol Biol Phys. 2008;71:330-7.

83. Talcott JA, Rossi C, Shipley WU, Clark JA, Slater JD, Niemierko $A$, et al. Patient-reported long-term outcomes after conventional and high-dose combined proton and photon radiation for early prostate cancer. JAMA. 2010;303:1046-53.

84. Babaian RJ, Donnelly B, Bahn D, et al. Best practice statement on cryosurgery for the treatment of localized prostate cancer. J Urol. 2008;180:1993-2004.

85. Warmuth M, Johansson T, Mad P. Systematic review of the efficacy and safety of high-intensity focussed ultrasound for the primary and salvage treatment of prostate cancer. Eur Urol. 2010;58:803-15.

86. Donnelly BJ, Saliken JC, Brasher PM, Ernst SD, Rewcastle JC, $\mathrm{Lau} \mathrm{H}$, et al. A randomized trial of external beam radiotherapy versus cryoablation in patients with localized prostate cancer. Cancer. 2010;116:323-30. 